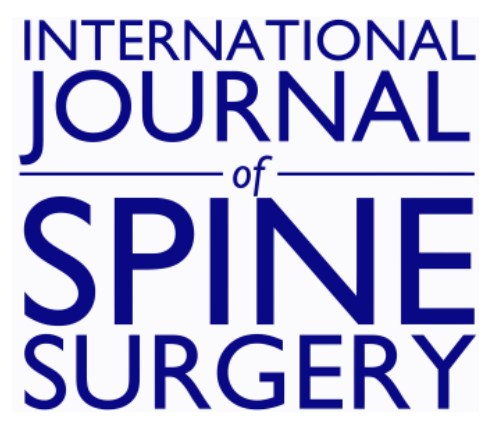

\title{
A Modified Triangular Osteosynthesis Protocol for the Rod and Pedicle Screw Fixation of Vertical Unstable Sacral Fractures
}

Dawei Tian, Xiaojun Guo, Na Liu, Bo Wang, Hongsheng He and Min Xiong

Int J Spine Surg 2021, 15 (3) 485-493

doi: https://doi.org/10.14444/8070

http://ijssurgery.com/content/15/3/485

This information is current as of April 25, 2023.

Email Alerts Receive free email-alerts when new articles cite this article. Sign up at:

http://ijssurgery.com/alerts

The International Journal of Spine Surgery

2397 Waterbury Circle, Suite 1,

Aurora, IL 60504, Phone: +1-630-375-1432 


\title{
A Modified Triangular Osteosynthesis Protocol for the Rod and Pedicle Screw Fixation of Vertical Unstable Sacral Fractures
}

\author{
DAWEI TIAN, MD, PHD,${ }^{1}$ XIAOJUN GUO, MD, ${ }^{2}$ NA LIU, MS, ${ }^{3}$ BO WANG, MD, PHD,${ }^{4}$ \\ HONGSHENG HE, MD, ${ }^{1}$ MIN XIONG, MD, PHD ${ }^{1}$ \\ ${ }^{I}$ Department of Orthopedics, Hubei University of Medicine Dongfeng Hospital, Shiyan 442008, Hubei, China, ${ }^{2}$ Department of General Surgery, Hubei \\ University of Medicine Dongfeng Hospital, Shiyan 442008, Hubei, China, ${ }^{3}$ Department of Information Management and Information System, Hubei University \\ of Medicine, Shiyan 442008, Hubei, China, ${ }^{4}$ Department of Spine Section, University of Chinese Academy of Sciences-Shenzhen Hospital, Shenzhen 518003 , \\ Guangdong, China
}

\begin{abstract}
Background: The stabilization of vertical unstable sacral fractures has been a complex problem that is a challenge to current standard approaches. Here, we attempted to evaluate a modified technique for sacral fractures.

Methods: In the modified triangular osteosynthesis technique, we adopted a vertical and transverse fixation with a rod and pedicle screw system to reduce and fix sacral fractures in 28 subjects. The postsurgery effect of this technique was evaluated by physical examination and radiography.

Results: In the postoperative day 1, the patients were able to move body position passively from the lateral side to supine and exercise their legs by themselves. After a follow up of 20 months, radiological evaluation showed that fracture fragment reduction was excellent in 18 (64\%), good (displacement 5-10 $\mathrm{mm}$ ) in $8(29 \%)$, and fair (displacement $10-15 \mathrm{~mm})$ in $2(7 \%)$ patients. Three patients with a preoperative perineal neurological impairment had a complete recovery after surgical decompression. All patients had achieved bone union of fractures, and no loss of fracture reduction was detected.

Conclusions: The modified procedures offered an easier approach to fix vertical unstable sacral fractures, thereby achieving quicker and stable functionality. This suggests an alternative approach to manage unstable sacral fractures.

Level of Evidence: 4.

Clinical Relevance: The stabilization of vertical unstable sacral fractures has been a complex problem that is a challenge to current standard approaches. We attempted to introduce a modified technique for sacral fractures.

Lumbar Spine

Keywords: triangular osteosynthesis, vertical unstable sacral fracture, a rod and pedicle screw fixation system
\end{abstract}

\section{INTRODUCTION}

Vertical unstable sacral fracture, part of Type C pelvic ring injuries, results in rotatory and vertical instability, frequently leading to morbidities or even mortality due to pain and malunion. ${ }^{1}$ To overcome these complications, an optional treatment is much urgently needed to reconstruct the spine-pelvic junction so that early weight bearing is allowed and easier care is possible, particularly for patients with multiple injuries. ${ }^{2}$

Operative reduction and internal fixation have been widely used for the treatment of vertical sacral fractures. However, the standard solution for posterior pelvic fixation options was to reconstruct plates by using multiple sacroiliac screws and sacral bars. This protocol suffers some disadvantages that may cause complications, despite some obvious advantages: the sacroiliac screws have gained popularity because of allowing minimally invasive and early definitive fixation, whereas iliosacral screws were used to reduce posterior pelvic ring injuries percutaneously. However, whether a surgery is successful by following this protocol is dependent on the fracture type, local situation, and posttraumatic timepoint. Of note, there were several limitations of iliosacral screw fixation, which requires fewer screws due to $\mathrm{S} 1$ body comminution, but fewer screws can compromise the screw function. For instance, it is impossible for a single iliosacral screw to fix and stabilize sagittal deformity. Additionally, it should be cautioned that, if iliosacral screw fixation must be used, iatrogenic 
nerve injury may be provoked due to compression and destruction of the neuroforaminal tunnels. Moreover, long-time traction before operation might cause to delay treatment, and beneficial results of subsequent recovery may be compromised. ${ }^{3}$ Despite various techniques assisted with computed tomography (CT)-guided/computer-navigated screw positioning were employed to increase precision of screw positioning, none of these interventions could directly counterbalance the biplanar force on the posterior pelvic ring, hence providing adequate fixation for an early unrestricted weight-bearing status.

Triangular osteosynthesis (TOS), initially developed by Schildhauer et al, is a modification of spinopelvic fixation (SPF) techniques. ${ }^{4-6}$ This fixation technique combines a lumbopelvic distraction osteosynthesis (the vertical component) with a transverse fixation (the horizontal component), ie, iliosacral screw osteosynthesis or tension-band transiliac plate osteosynthesis. A series of clinical and biomechanical studies have demonstrated that TOS had offered sufficient multiplanar stability and satisfactory fixation. By doing this way, the surgery support can counterbalance the force on the posterior pelvic ring, making it possible for patients to have early weight bearing. Based on the principles of TOS, we described a modified TOS protocol to reduce and to fix vertical unstable sacral fractures. It consists of vertical and transverse fixation with a rod and pedicle screw system. In this report, we investigated the clinical outcomes by radiology and examined the reduction and fixation of vertical unstable sacral fractures. We further evaluated the biomechanical function after definitive reduction and stabilization as well as the surgery-related complications.

\section{MATERIALS AND METHODS}

\section{Recruitment of Patients}

From October 2010 to July 2016, 28 patients with vertical unstable sacral fractures were recruited (male, 16; female, 12; median age of 30 [16-59] years; Institutional Review Board Approval No. 201008002; Clinical Research Registration No: ISRCTN81077281; Table 1). Fifteen of them (15/ 28) were hemodynamically unstable. All patients (28/28) had symphyseal ruptures or pubic rami fractures; 16 of them (16/28) had fractures of the lower extremities as well. Three of them $(3 / 28)$ appeared to have perineal neurological deficit. According to the AO Foundation/Orthopaedic Trauma Association classification, there were 10 $\mathrm{C} 1$ injuries and $18 \mathrm{C} 2$ injuries; based on Denis classification, $^{7}$ there were 7 Zone I injuries and 20 Zone II injuries (Table 1). Our work was compliant with the Strengthening the Reporting of Observational Studies in Epidemiology criteria. ${ }^{8} \mathrm{X}$-ray trauma series (AP [anterior-posterior], inlet, and outlet of pelvis), pelvic CT and 3-dimensional CT evaluation were performed at the time of emergency admission throughout all follow-up evaluations.

\section{Surgical Managements}

According to trauma resuscitation protocol, hemodynamically unstable patients had initially undergone an anterior external fixation in a supine position. For hemodynamically stable patients with a major vertical displacement, distal femoral traction was performed. When a patient's condition was sufficiently improved to allow surgical intervention, a definitive surgery convened, and the prior external fixation could be removed. Treatment of posterior pelvis injury started with reduction and stabilization; a patient was placed in the prone position, assisted with an image boost (conventional C-arm fluoroscopy, Shimadzu, Japan). The surgery was performed by 1 surgeon, and a median lag time between initial trauma and definite operation was around 6 (1-17) days.

Next, 2 initial incisions were made for a subject, and this applies to all patients. A longitudinal and curved approach to the lumbosacral spine was made on a posterior median line that lays between the L4, L5, and iliac bone through the posterior-superioriliac-spine (PSIS) and is parallel to the sacroiliac joint; the other one was made along the contralateral iliac bone through PSIS. We then performed thoracolumbar fascia and paravertebral muscle mass splitting to expose L4, L5, sacroiliac joint, and bilateral iliac bone through PSIS. To allow a deeper seating of the screw head and to prevent prominence of the metal work under the soft tissues, 2 bone windows were made by a reamer on each side at the bilateral iliac bones. A third paravertebral incision was made to perform sacral laminectomy for patients with preoperative perineal neurological impairment.

We adopted a universal spinal system (USS) rod and pedicle screw system (USS Fixateur Interne; Synthes USA) during the procedure. First, pedicle screw insertion points were identified. After tapping, 
Table 1. Preoperation patient data.

\begin{tabular}{|c|c|c|c|c|c|c|c|}
\hline Patient & Age, $y$ & Sex & Cause & $\begin{array}{c}\text { Preoperative } \\
\text { Neurological Deficit }\end{array}$ & $\begin{array}{l}\text { Fracture } \\
\text { Zone }\end{array}$ & $\begin{array}{l}\text { Fracture } \\
\text { Type }\end{array}$ & Associated Lesion \\
\hline 1 & 32 & Male & Car accident & No & II & $\mathrm{C} 1$ & No \\
\hline 2 & 16 & Female & Suicidal jump & Yes & II & $\mathrm{C} 2$ & Right femur fracture \\
\hline 3 & 41 & Female & Car accident & No & I & $\mathrm{C} 1$ & Right pilon fracture \\
\hline 4 & 25 & Male & Car accident & No & II & $\mathrm{C} 2$ & Right tibial fracture \\
\hline 5 & 38 & Male & Suicidal jump & No & II & $\mathrm{C} 1$ & Left pilon fracture \\
\hline 6 & 40 & Male & Car accident & No & II & $\mathrm{C} 2$ & No \\
\hline 7 & 26 & Female & Car accident & No & II & $\mathrm{C} 2$ & No \\
\hline 8 & 32 & Male & Suicidal jump & No & I & $\mathrm{C} 2$ & Left femur fracture \\
\hline 9 & 59 & Female & Car accident & No & II & $\mathrm{C} 2$ & No \\
\hline 10 & 28 & Female & Suicidal jump & Yes & II & $\mathrm{C} 2$ & Right calcaneus fracture \\
\hline 11 & 33 & Male & Car accident & No & II & $\mathrm{C} 2$ & No \\
\hline 12 & 31 & Male & Car accident & No & II & $\mathrm{C} 1$ & Left femur fracture \\
\hline 13 & 33 & Female & Car accident & No & II & $\mathrm{C} 2$ & No \\
\hline 14 & 29 & Female & Car accident & No & II & $\mathrm{C} 2$ & Left tibial fracture \\
\hline 15 & 52 & Female & Fall & No & I & $\mathrm{C} 1$ & Left femur fracture \\
\hline 16 & 28 & Female & Car accident & No & II & $\mathrm{C} 2$ & No \\
\hline 17 & 25 & Male & Fall & Yes & II & $\mathrm{C} 2$ & Right pilon fracture \\
\hline 18 & 42 & Male & Car accident & No & II & $\mathrm{C} 2$ & No \\
\hline 19 & 21 & Female & Car accident & No & I & $\mathrm{C} 1$ & Right femur fracture \\
\hline 20 & 33 & Male & Fall & No & II & $\mathrm{C} 2$ & Right calcaneus fracture \\
\hline 21 & 52 & Male & Car accident & No & II & $\mathrm{C} 2$ & Right and left femur fracture \\
\hline 22 & 43 & Female & Car accident & No & II & $\mathrm{C} 2$ & No \\
\hline 23 & 37 & Male & Car accident & No & II & $\mathrm{C} 2$ & Left femur fracture \\
\hline 24 & 26 & Male & Fall & No & I & $\mathrm{C} 1$ & Left tibial fracture \\
\hline 25 & 31 & Male & Car accident & No & I & $\mathrm{C} 1$ & No \\
\hline 26 & 22 & Male & Fall & No & II & $\mathrm{C} 2$ & Right tibial fracture \\
\hline 27 & 19 & Female & Car accident & No & II & $\mathrm{C} 1$ & No \\
\hline 28 & 52 & Male & Car accident & No & I & $\mathrm{C} 1$ & No \\
\hline
\end{tabular}

2 pedicle screws were inserted into the pedicle of L4 and L5. Another 3 pedicle screws were inserted into the ilium between the inner and outer cortices as an iliac screw. Ilium screw size was determined based on the distance from the recessed entrance of PIIS along sciatic buttress to the ending of the anterior inferior iliac spine, ranging from 60 to $110 \mathrm{~mm}$. Then a straight rod was precurved into an $\mathrm{L}$ shape with the angle according to screws position in L4, L5 pedicle, and iliac bone through the PSIS that consisted of a vertical fixation; the rod was connected with the iliac bone through the PSIS to finalize a vertical fixation. This was also connected in situ with bilateral parallel iliac screws through the PSIS to achieve a transverse fixation. To restore fractures into their original states, screws were used as "joysticks," and a manipulator between the 2 screws was used. By doing so, we either loosened or tightened 1 screw alternatively by using a compressor to perform the reduction. Meanwhile, manual forces were gently used to distract the ilium both posteriorly and caudally. The correct reduction of sacral fractures was confirmed posteriorly by palpation of the symmetrical position of bilateral PSIS and the image boost. Lastly, the pedicle and iliac screw were connected sitting with the rod system (Figure 1). ${ }^{4,9}$
After copious irrigation, the surgical wound was closed with 2 suction drainages. Next, in a supine position, a dynamic compression plate was used to fix the disrupted symphysis pubis or fractures of the pelvic rami. This fixation was carried out depending on displacement level of anterior pelvic ring injury.

\section{Postoperative Management}

Postoperative management included bed rest, analgesics, and broad-spectrum antibiotics. A lumbosacral corset was used to assist a subject to move and exercise in a progressive way depending on the subject's tolerance. This was to mobilize the patients to recover motor function and prevent immobilization-related complications such as deep venous thrombosis and urinary infections. On the postoperative day 1, all patients could move their body position passively from the lateral side to supine, and the patients could exercise lower extremities by themselves. Depending on the pain level of a patient, they were encouraged to ride in a wheelchair or to walk under partial weight bearing with a walker or crutch. Patients were encouraged to walk for 30 minutes with a walker if bone regrowth was revealed and there was no pelvic pain or any instable complaint. Once bone union of the fracture was complete, the fixation devices were removed to 

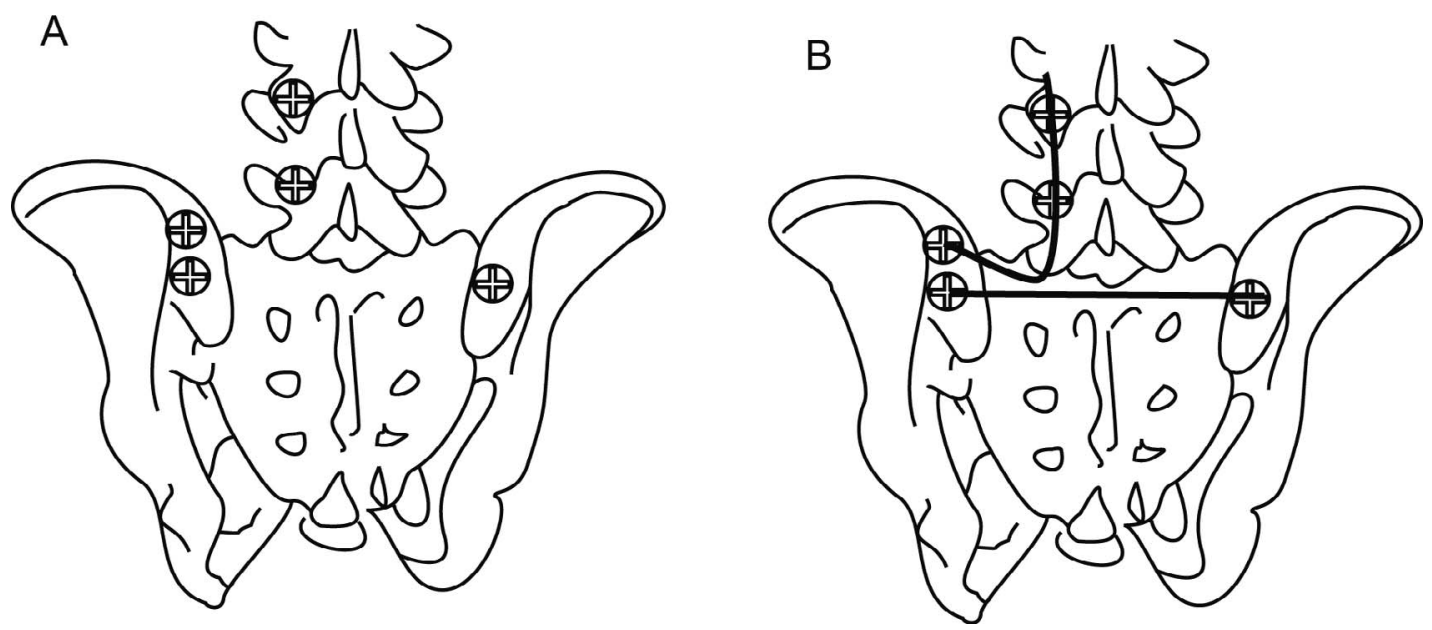

Figure 1. Diagram demonstrating posteroanterior view and the technique of fixation: (A) the point of pedicle insertion hole in L4, L5, and ilium, (B) poly-axial screws in L4, L5, and ilium.

avoid potential metal breakage from increased articular movements of the sacroiliac joint during full weight bearing. This proactive procedure can also prevent possible spontaneous fusion in the lower lumbar spine or the prominent tulips of the iliac screws from provoking pain and disc failure in young active adults. It can also prevent infection and prominence of the tulips, as the prominent tulips of the iliac screws provoke pain. Late displacement of the posterior pelvis fracture was measured based on x-ray imaging (AP, inlet, outlet views) and $\mathrm{CT}$; residual postoperative displacement was evaluated by an independent observer.

\section{RESULTS}

All 28 subjects were followed up for an average of 20 (16-32) months with routine visits occurring at 1 , $3,6,12,24$, and 32 months for physical and radiographic examinations. An average operation time for this procedure was 175 (118-240) minutes, and the average amount of intraoperative blood loss was 1643 (950-2320 mL; Table 2). No patients died during operation or afterwards. For the patients with neurologic impairment, surgical decompression was performed via sacral laminectomy. Intra-operative findings revealed bony retropulsion with compression of nerve roots S1 and S2 and hematoma in the spinal canal, without dural tears. However, the spinal cord was intact, and there was no injury of neural structures. After removal of the fracture fragments and hematoma, a slow but complete recovery was accomplished. Iatrogenic lesion was not observed. Superficial infection was reported in 4 cases, and this was treated with surgical debridement and local antibiotics. The wound was recovered in 4 weeks; so was the fracture union in 5 months.

Two patients had complaints related to distal screws inserted in the region of the PSIS; the tenderness disappeared after implant removal within 10 months. No skin lesions were observed for both cases. Loosening of the hardware occurred in 1 case in the fifth month after operation, but the fracture had healed; loss of reduction and metal breakage were not observed.

Table 2. Operation data.

\begin{tabular}{|c|c|c|c|c|}
\hline Patient & $\begin{array}{c}\text { Delay to } \\
\text { Surgery, d }\end{array}$ & $\begin{array}{l}\text { Duration of } \\
\text { Surgery, min }\end{array}$ & $\begin{array}{c}\text { Loss of } \\
\text { Blood, mL }\end{array}$ & $\begin{array}{c}\text { Vessel and } \\
\text { Nerve Injuries }\end{array}$ \\
\hline 1 & 12 & 125 & 1021 & No \\
\hline 2 & 1 & 240 & 2100 & No \\
\hline 3 & 4 & 145 & 950 & No \\
\hline 4 & 8 & 153 & 1230 & No \\
\hline 5 & 10 & 180 & 1850 & No \\
\hline 6 & 14 & 150 & 1390 & No \\
\hline 7 & 17 & 195 & 2020 & No \\
\hline 8 & 3 & 182 & 1500 & No \\
\hline 9 & 7 & 210 & 1700 & No \\
\hline 10 & 2 & 136 & 1327 & No \\
\hline 11 & 5 & 203 & 1750 & No \\
\hline 12 & 7 & 131 & 1265 & No \\
\hline 13 & 1 & 165 & 1504 & No \\
\hline 14 & 6 & 210 & 1980 & No \\
\hline 15 & 10 & 230 & 2105 & No \\
\hline 16 & 3 & 153 & 1065 & No \\
\hline 17 & 8 & 185 & 1740 & No \\
\hline 18 & 2 & 132 & 1548 & No \\
\hline 19 & 4 & 172 & 1806 & No \\
\hline 20 & 6 & 200 & 1850 & No \\
\hline 21 & 3 & 139 & 1536 & No \\
\hline 22 & 8 & 205 & 2106 & No \\
\hline 23 & 9 & 210 & 2008 & No \\
\hline 24 & 11 & 226 & 2305 & No \\
\hline 25 & 5 & 140 & 1725 & No \\
\hline 26 & 12 & 236 & 2320 & No \\
\hline 27 & 7 & 118 & 1206 & No \\
\hline 28 & 5 & 125 & 1106 & No \\
\hline
\end{tabular}


Table 3. Postoperation patient data.

\begin{tabular}{lc}
\hline Parameter & Value \\
\hline No. patients & 28 \\
Follow-up, mean (range), mo & $20(16-32)$ \\
Postoperative neurological deficit & 0 \\
Franck tenderness over distal screws & 2 \\
Skin lesion & 0 \\
Deep infection & 0 \\
Loss of reduction & 0 \\
Fracture heal & 28 \\
Implant loss & 1 \\
Implant removal, mean (range), mo & $13.5(10.5-18.1)$ \\
\hline
\end{tabular}

According to the Nelson and Duwelius standard, ${ }^{10}$ radiological evaluations showed fracture fragment reduction was excellent (displacement 0 $5 \mathrm{~mm}$ ) in $18(64 \%)$, good (displacement $5-10 \mathrm{~mm}$ ) in $8(29 \%)$, and fair (displacement $10-15 \mathrm{~mm}$ ) in 2 of the subjects $(7 \%)$, respectively. Vertical reduction was excellent in 15 patients $(54 \%)$, good in 10 $(36 \%)$, and fair in 3 of the cases $(10 \%)$. A displacement more than $15 \mathrm{~mm}$ was not observed. The fracture union was evaluated by x-ray and CT by examination of the presence of callus formation. The mean time of fracture healing was 4 (3-6) months. The implant was removed at a mean of 13.5 (10.5-18.1) months (Table 3). There was no additional displacement at the time of the last follow up compared with the immediate postoperative state (Figures 2 and 3).

All the patients were able to sit, lie on their sides, and stand with a crutch after operation. Fifteen patients were not able to put full weight on the operated extremity (mean $26 \pm 5$ weeks), partially due to concomitant injuries. Thirteen patients without concomitant injuries were able to bear full weight after $15 \pm 2$ weeks. All patients showed normal walking capability at final follow-up evaluation. No apparent motor weakness was detected in any of the patients. Two patients reported mild pain on gait that was located at the low back region; however, they did not need pain medication. Regarding the working status at the final followup evaluation, 24 patients $(86 \%)$ returned to their previous job, whereas 4 of them $(14 \%)$ were unable to return to a similar job. According to the criteria of Rommens and Hessmann, ${ }^{11}$ the clinical results showed $19(68 \%)$ excellent cases, $7(25 \%)$ good, and $2(7 \%)$ moderate ones.

\section{DISCUSSION}

The sacrum is a critical link for weight of the trunk to be transmitted to the legs. It is a location of frequently complained-about back pain. Fixation of sacral fractures has been challenging for local anatomic complexities, unique biomechanical forces, and poor bone quality. ${ }^{4,12}$

Previous biomechanical analysis has confirmed that TOS was among the most reliable methods for stable posterior pelvic fixation. ${ }^{13-16}$ This fixation technique combined a lumbopelvic distraction osteosynthesis with a transverse fixation. The transiliac internal fixator, one of the transverse fixations, provides the same biomechanical stability as other implants tested. There are several alternative ways to use this device; however, the modified protocol we demonstrated here had 2 distinct elements: (1) rod and pedicle screw fixation was chosen as reduction and fixation for TOS; (2) a transiliac internal fixator was applied as transverse fixation.

The TOS construct bypassed the sacrum and sacroiliac joint and unloaded the area of injury by mimicking a normal load transfer from acetabulae through the sacroiliac joints to the lumbar spine, providing a good level of protection against cranial migration of the injured pelvic ring. ${ }^{16,17}$ The TOS provided greater immediate postoperative stability and prevented loss of reduction in comminuted vertical shear pelvic fracture. The reliability of this fixation is justifiable as it allows earlier full weight bearing (as early as 6 weeks postoperatively) without a need for cumbersome external braces. This configuration also allows comprehensive decompression of sacral neural elements and likely reduction of sacral fractures. ${ }^{9,18}$ In general, it is particularly suited for the treatment of sacral fractures.

TOS fixation instruments were reconstruction plates and multiple screws as well as sacroiliac screws, sacral bars, and a rod and pedicle screw system. These instruments can be used to fix limited complications but are not useful to do fracture reduction. For example, plate and screws used for fixation usually work well when prior fracture reduction has been successful made. However, by following TOS protocol, an excellent reduction and fixation can be accomplished altogether to treat fractures. The rod and pedicle can offer both vertical and transverse reduction and fixation. Given that TOS is a complicated major surgery, incidence of soft tissue damages (wound infection, dehiscence, and subcutaneous necrosis) was higher than a vertical or transverse fixation alone, especially when transsacral plating was performed for horizontal pelvic ring stabilization. ${ }^{19}$ 


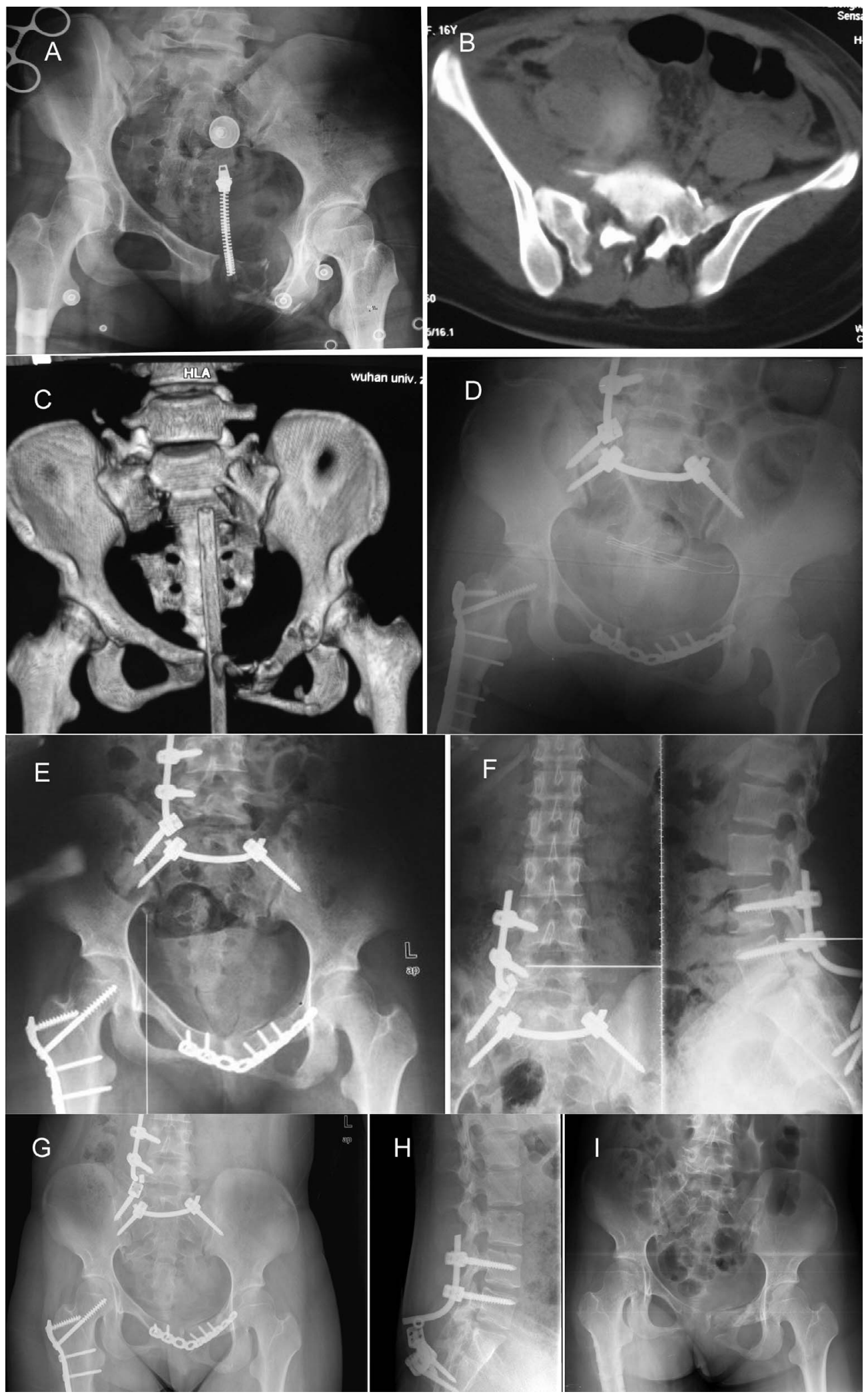

Figure 2. (A)-(C) Radiographs of a 16-year-old girl with right vertical unstable sacral fractures and right femur fractures, showing a preoperation anteroposterior $x$ ray, computed tomography (CT) coronal and 3-dimensional view and (D) the immediate postoperative scans after reduction and internal fixation. (E). Postoperative anteroposterior x-ray at the second month. (F) Loosening of the hardware was detected at the fifth month. (G) $-(\mathrm{H})$ A follow-up view at the 10th month. (I) The anteroposterior $x$-ray at the 11 th month indicates hardware removal; no loss of reduction was detected. 

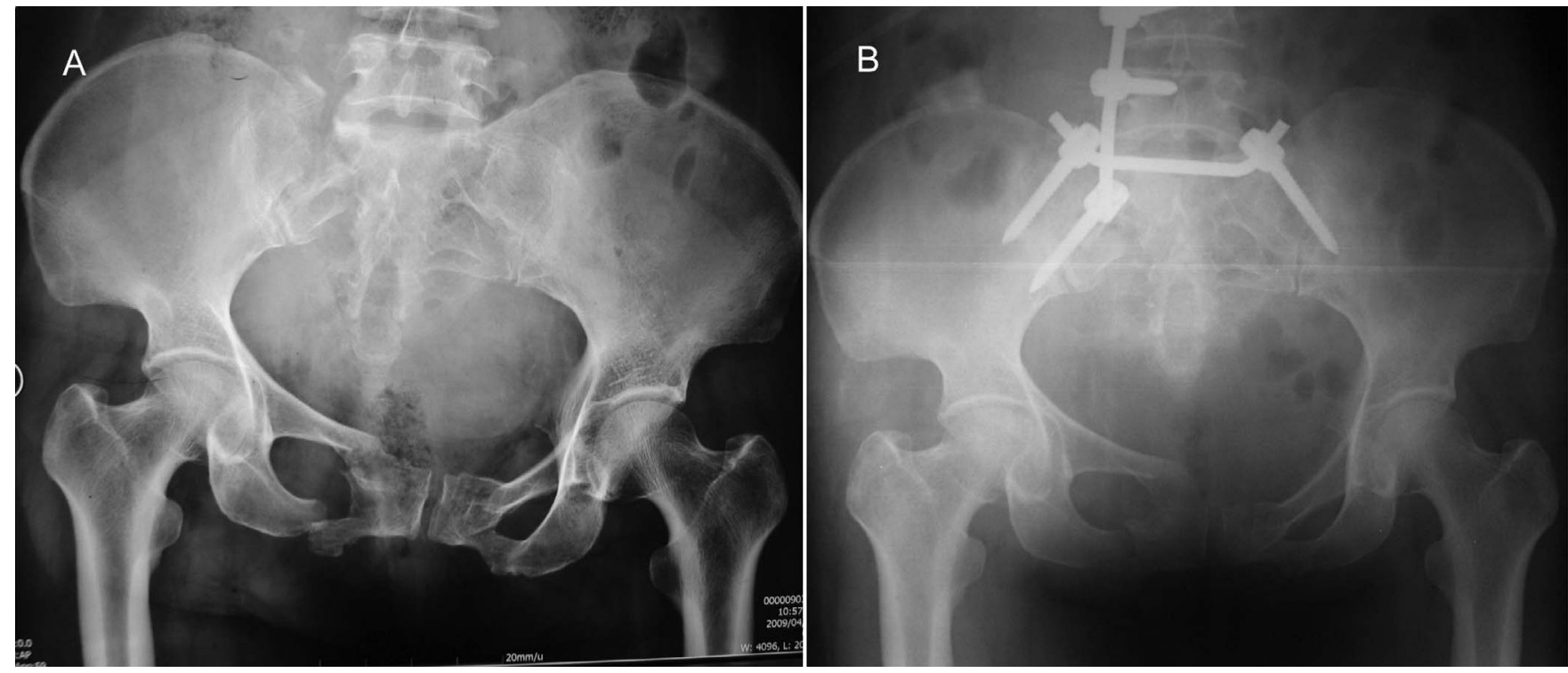

Figure 3. Radiographs of a 59-year-old female with right vertical unstable sacral fractures before and after operation. (A) An initial anteroposterior scan. (B) An immediate postoperative scan after reduction and posterior fixation.

The pedicle screw system has been developed as a procedure for posterior internal fixation of the thoracic, lumbar, and lumbosacral spines. Pull-out strength is related to screw-cortical bone interface, as well as the screw length and width. Some pedicle and iliac screw hardware provide only partially threaded iliac screws with limited length, whereas polyaxial pedicle screws with lower prominent screw head, optimal screw length, and width are better options for lumbopelvic reduction and fixation. We proposed a low-profile side opening system. With the help of a USS, our approach could determine an optimal screw length to support the main stress of vertical and rotational shear. This applies sound biomechanical force to achieve compression and makes the force close to the body gravity center. The use of our 3dimensional variable connection system can also provide an easier, correct reduction and fixation. After all, the current connection system allows an immediate, progressive weight bearing and safe nursing even in a case with multiple traumas. Specifically, given these obvious advantages, osteosynthesis of the anterior pelvic ring may not be necessary, as osteosynthesis may only work well for some cases depending on displacement level of anterior pelvic ring injury.

Additionally, we have demonstrated that a visualized, progressive vertical reduction with precision can be easily managed with our USS system. First, the connecting rod of the USS system is fixed to 1 pedicle screw and set loosely on the second one. With the pedicle screw used as a joystick, a surgeon could change the direction and bridging-fixation range reversibly between the sacrum and the ilium, while the vertical unstable sacral fractures could be compressed or distracted directly. By doing so, the fractures can be reduced both vertically and horizontally. Once a correct reduction is achieved, the connecting rod is secured to the second pedicle screw, making it possible to fix fractures with the rod being adjusted in 3 dimensions. The posterior surgical management allows a nerve decompression, which is important for vertically unstable sacral fractures with neurological insult. ${ }^{20}$

There are 2 potential challenges in the rod and screw system. Skin breakdown over the sacroiliac joint is a concern that may be caused by protruded rod-iliac screw connection. The skin overlying the sacroiliac joint lacks underlying muscle tissues, but pedicle screws had high prominence, which tended to develop a pressure sore when a patient is in supine position. Saiki et al reported a high incidence of skin problems over this site after the use of rod and pedicle screw internal fixation devices. ${ }^{21}$ To avoid such a complication, a bone window should be made, lower prominence pedicle screws should be chosen, and the connecting device at this site should be buried in the iliac cortex. Another problem is the difficulty to bend a rod into an $\mathrm{L}$ shape to fit screw positions at L4, L5, and the iliac bone throughout PSIS. In this situation, a curved individual rod based on 
imaging scans may be developed before operation. This bending is tricky as great bending may weaken the rod to resist against axial and rotational forces.

\section{CONCLUSIONS}

In conclusion, vertical unstable sacral fractures can be successfully reduced and fixed in a correct position using a rod and pedicle screw system between the L4, L5 vertebra, and the ilium. Our surgical procedure can easily and reversibly reduce and fix the vertical unstable sacral fractures with a good reconstruction of pelvic ring, thus providing a great immediate postoperative stability. This procedure is a suitable alternative that may be useful in treating vertical unstable sacral fractures.

\section{ACKNOWLEDGMENTS}

This research was supported in part by the Jingchu Program for Excellent Talents, the Hubei Provincial Department of Education (No. 54, 2017), Hubei Province Health and Family Planning Scientific Research Project (WJ2019F069), and Hubei Natural Science Foundation (2020CFB582).

\section{REFERENCES}

1. Tile M. Pelvic ring fractures: should they be fixed? J Bone Joint Surg Br. 1988;70(1):1-12.

2. Mouhsine E, Wettstein M, Schizas C, et al. Modified triangular posterior osteosynthesis of unstable sacrum fracture. Eur Spine J. 2006;15(6):857-863.

3. Dawei T, Na L, Jun L, et al. A novel fixation system for sacroiliac dislocation fracture: internal fixation system design and biomechanics analysis. Clin Biomech (Bristol, Avon). 2013;28(2):129-133.

4. Schildhauer TA, Josten C, Muhr G. Triangular osteosynthesis of vertically unstable sacrum fractures: a new concept allowing early weight-bearing. J Orthop Trauma. 2006;20(1):S44 51.

5. Schildhauer TA, Ledoux WR, Chapman JR, et al. Triangular osteosynthesis and iliosacral screw fixation for unstable sacral fractures: a cadaveric and biomechanical evaluation under cyclic loads. J Orthop Trauma. 2003;17(1):22-31.

6. Kach K, Trentz O. [Distraction spondylodesis of the sacrum in "vertical shear lesions" of the pelvis]. Unfallchirurg. 1994;97(1):28-38.

7. Denis F, Davis S, Comfort T. Sacral fractures: an important problem. Retrospective analysis of 236 cases. Clin Orthop Relat Res. 1988;227:67-81.

8. von Elm E, Altman DG, Egger $M$, et al. The Strengthening the Reporting of Observational Studies in
Epidemiology (STROBE) statement: guidelines for reporting observational studies. Int J Surg. 2014;12(12):1495-1499.

9. Schildhauer TA, Bellabarba C, Nork SE, et al. Decompression and lumbopelvic fixation for sacral fracturedislocations with spino-pelvic dissociation. J Orthop Trauma. 2006;20(7):447-457.

10. Nelson DW, Duwelius PJ. CT-guided fixation of sacral fractures and sacroiliac joint disruptions. Radiology. 1991;180(2):527-532.

11. Rommens PM, Hessmann MH. Staged reconstruction of pelvic ring disruption: differences in morbidity, mortality, radiologic results, and functional outcomes between B1, B2/ B3, and C-type lesions. J Orthop Trauma. 2002;16(2):92-98.

12. Jones CB, Sietsema DL, Hoffmann MF. Can lumbopelvic fixation salvage unstable complex sacral fractures? Clin Orthop Relat Res. 2012;470(8):2132-2141.

13. Dienstknecht T, Berner A, Lenich A, et al. Biomechanical analysis of a transiliac internal fixator. Int Orthop. 2011;35(12):1863-1868.

14. Tis JE, Helgeson M, Lehman RA, et al. A biomechanical comparison of different types of lumbopelvic fixation. Spine (Phila Pa 1976). 2009;34(24):E866-872.

15. Yu BS, Zhuang XM, Zheng ZM, et al. Biomechanical advantages of dual over single iliac screws in lumbo-iliac fixation construct. Eur Spine J. 2010;19(7):1121-1128.

16. Sagi HC. Technical aspects and recommended treatment algorithms in triangular osteosynthesis and spinopelvic fixation for vertical shear transforaminal sacral fractures. $J$ Orthop Trauma. 2009;23(5):354-360.

17. Sagi HC, Militano U, Caron T, et al. A comprehensive analysis with minimum 1-year follow-up of vertically unstable transforaminal sacral fractures treated with triangular osteosynthesis. J Orthop Trauma. 2009;23(5):313-319; discussion 319-321.

18. Ayoub MA. Vertically unstable sacral fractures with neurological insult: outcomes of surgical decompression and reconstruction plate internal fixation. Int Orthop. 2009;33(1):261267.

19. Schildhauer TA, Josten C, Muhr G. Triangular osteosynthesis of vertically unstable sacrum fractures: a new concept allowing early weight-bearing. J Orthop Trauma. 1998;12(5):307-314.

20. Zelle BA, Gruen GS, Hunt T, et al. Sacral fractures with neurological injury: is early decompression beneficial? Int Orthop. 2004;28(4):244-251.

21. Saiki K, Hirabayashi S, Horie T, et al. Anatomically correct reduction and fixation of a Tile $\mathrm{C}-1$ type unilateral sacroiliac disruption using a rod and pedicle screw system between the $\mathrm{S} 1$ vertebra and the ilium: experimental and clinical case report. J Orthop Sci. 2002;7(5):581-586.

Disclosures and COI: Dawei Tian and Xiaojun Guo contributed equally to this paper. The authors declare that they have no conflict of interests. This study was approved by the Research Review Board of SinoPharm Dongfeng General Hospital (Institutional Review Board Approval No. 201008002) and is listed in the ISRCTN registry (No. ISRCTN81077281). DT, XG, and MX designed the study; DT and XG performed the surgery; DT and XG collected and analyzed the 
data; DT, XG, and MX drafted the manuscript. $\mathrm{NL}, \mathrm{BW}$, and $\mathrm{HH}$ assisted with the preparation of surgery and participated in drafting the manuscript. All authors read and approved the final manuscript.

Corresponding Authors: Min Xiong, MD, PhD, Department of Orthopedics, Hubei University of Medicine Dongfeng Hospital, Shiyan 442008, Hubei, China. Phone: 0086 (719) 827-2448; Fax: 0086 (719) 827-2448; Email: minxiong1975@yahoo. com. Dawei Tian, MD, PhD, Department of Orthopedics, Dongfeng Hospital. Email: davidiswho@sina.com.

Published 1 June 2021

This manuscript is generously published free of charge by ISASS, the International Society for the Advancement of Spine Surgery. Copyright (C) 2021 ISASS. To see more or order reprints or permissions, see http://ijssurgery.com. 